Perception of nonlinear distortion on emulation of frequency responses of headphones

Pablo Gutierrez-Parera, and Jose J. Lopez

Citation: The Journal of the Acoustical Society of America 143, 2085 (2018); doi: 10.1121/1.5031030

View online: https://doi.org/10.1121/1.5031030

View Table of Contents: http://asa.scitation.org/toc/jas/143/4

Published by the Acoustical Society of America

Copyright (2018) Acoustical Society of America. This article may be downloaded for personal use only. Any other use requires prior permission of the author and the Acoustical Society of America.

The following article appeared in "The Journal of the Acoustical Society of America 143, 2085 (2018); doi: 10.1121/1.5031030" and may be found at https://asa.scitation.org/doi/10.1121/1.5031030. 


\title{
Perception of nonlinear distortion on emulation of frequency responses of headphones $(\mathrm{L})$
}

\author{
Pablo Gutierrez-Parera ${ }^{\text {a) }}$ and Jose J. Lopez \\ ITEAM Institute, Universitat Politècnica de València, Valencia, 46022, Spain
}

(Received 15 December 2017; revised 27 February 2018; accepted 26 March 2018; published online 16 April 2018)

The equalization of headphones can force transducers to work in a non-linear condition, producing non-linear distortion. Depending on the headphone model and the reproduction level, that distortion can be audible. In this study, headphones of diverse quality and price were compelled to emulate the same target frequency response and the non-linear distortion was measured. A Diagonal Volterra model was used to simulate the different headphones with and without distortion. A perceptual test was carried out to determine the level of reproduction above which non-linear distortion is perceived for each headphone model. High correlation has been found between the level of detected distortion and retail prices of headphones. (C) 2018 Acoustical Society of America.

https://doi.org/10.1121/1.5031030

[JFL]

Pages: $2085-2088$

\section{INTRODUCTION}

In recent years there has been an increased interest in headphone listening. The advances in virtual and augmented reality can also boost the development of headphone technology and new techniques are appearing for headphone products. A clear possibility to enhance the perceived quality of a headphone is the use of active signal processing. Previous works suggest that frequency response is a dominant factor in the perceived quality; ${ }^{1,2}$ this fact has focused the studies of subjective user preferences on different target frequency responses. ${ }^{3}$ Some recent headphones and software for headphones put attention on the individualization of the listening experience by shaping or tailoring the frequency response., ${ }^{4,5}$ These works and new products are showing that with precise measurements and equalization, it is also possible to mimic the frequency response of a specific headphone with a different headphone model. ${ }^{3,6} \mathrm{~A}$ virtual headphone emulation has some limitations, as transducers could not have the capacity to reproduce specific frequencies; for example, the lowest or highest frequencies cannot be reachable for certain consumer headphone models. Besides, a strong equalization can force a transducer to work out of its linear condition, creating nonlinear distortion effects. Despite non-linearities being difficult to perceive due to masking effects, if some equalization is applied, audible distortion can be greatly increased, which can degrade the final perceived quality.

The objective of this work is to examine the perception of non-linear distortion when applying a target frequency response over different consumer headphone models. To this end, we have combined a simulation of the non-linear distortion measured in each of the headphone models, together with a virtual headphone listening test methodology. The frequency response of a high quality headphone should be emulated through a set of different headphones with quite different qualities. The distortion produced by each headphone must

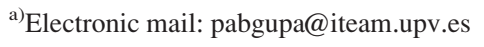

then be measured, along with the linear response obtained. With these parameters, a virtual headphone listening test has been performed, comparing samples of the headphone emulation with and without non-linear distortion. A much simpler test included in a previous work of the authors, which considered just direct non-linear distortion at one reproduction level, ${ }^{7}$ motivated this more in-depth and rigorous study.

\section{METHODS}

All the measurements of the headphones and the reproduction of sounds were done with a Head and Torso Simulator (HATS) model B\&K type 4100 and a MOTU Traveler sound card. A Sennheiser HD800 was chosen as the reference headphone because of its high quality, using its frequency response as the target to be emulated in the different headphones under test. Besides, this reference headphone was used to perform the virtual listening test. To emulate the target frequency response, 11 headphones of different qualities and prices were selected. They were intended to cover a wide range of possible common uses.

\section{A. Calibrated measurements of impulse responses with non-linear distortions after target response correction}

Non-linear distortion depends on the signal level applied to the headphones at each frequency. For this reason, all the headphone measurements carried out in this work have passed a calibration process. The calibration takes into account all the measure chain, including the HATS microphones sensitivity $(\mathrm{mV} / \mathrm{Pa})$ and the electrical full scale value (mVFS) of the sound card. The IEC $61672-1^{8}$ recommendation for A weighting pressure levels was employed.

In the first stage, the linear responses of the headphones were measured and a filter was calculated to model the target response. A logarithmic sweep of $5 \mathrm{~s}$ was used, covering 20 to $20000 \mathrm{~Hz}$ without pre-ringing effects. Both left and right transducers were measured with a sampling rate of $48 \mathrm{kHz}$. The following steps were implemented to obtain filters that 
emulate the target response over each tested headphone: (1) frequency response smoothing of $1 / 6$ octave for every headphone. (2) Calculation of the headphone's inverse filter by direct inversion of the measured response, from 20 to $20000 \mathrm{~Hz}$. (3) Spectral product of the inverted frequency response of the tested headphone and the target frequency response. (4) Limitation of the filter gain to $+20 \mathrm{~dB}$ to avoid excessive boost at certain frequencies. (5) Calculation of the equivalent minimum phase filter.

In the second stage, the individual target response filters were applied over each headphone and the non-linear distortions generated by these response corrections were measured. This is the key point of this work because it allows for the evaluation of the behavior of a poor quality response headphone in terms of distortion when it is corrected by equalization. The steps to accomplish these measurements were: (1) the synchronized swept-sine ${ }^{9}$ employed for measurement is filtered by the equalization filter for each headphone. (2) The resulting test signal is reproduced for each headphone at six different calibrated reproduction levels of $70,80,85,90,95$, and $100 \mathrm{dBA}$. These levels have been calibrated in the HATS ears for $20-20000 \mathrm{~Hz}$ pink noise. (3) With the recorded responses, second and third-order nonlinear distortions generated by the headphone were computed using the method described in Ref. 9.

Following this procedure, each of the tested headphones was forced to try to emulate the same target frequency response at the same acoustic pressure levels, which means that they were compelled to work in the same conditions. Figure 1 shows the linear magnitude responses achieved for each headphone after the equalization and the first two harmonics of distortion generated. To represent the second and third distortion orders for the six reproduction levels clearly at a glance, we have drawn them as two shaded areas where the lowest limit corresponds to $70 \mathrm{dBA}$ and the highest to $100 \mathrm{dBA}$; therefore, intermediate levels fall into the shaded area. The linear responses were almost identical for all six reproduction levels, so the mean is represented.

Reposition of the headphones is a convenient procedure for the frequency response measurements. ${ }^{10}$ However, the described measurements were done without reposition. In this experiment, we want to focus on the distortion generated during the emulation of a target frequency response; therefore, the correction of a mean frequency response would generate an unreliable measure of the distortion, as the final measurement of the emulated response with its distortion also depends on a fixed position. As the subjective test performed with these measures concentrates in the perception of the distortion, the possible variations in the linear frequency response were considered not relevant here.

\section{B. Virtual simulation of the non-linear distortion}

To simulate the non-linear distortion of each headphone obtained after the equalization, we used a Diagonal Volterra kernels model and a series of linear convolutions as described in Ref. 11. With this method, the transfer function of a non-linear system is estimated by means of a truncated Volterra series. The output signal of a non-linear system can be represented as an infinite sum of convolutions of the Volterra kernels with power series of the input signal. These Diagonal Volterra kernels are computed as a linear combination of each of the infinite orders of distortion impulse responses.

The distortion produced by headphones is in general low and decreases rapidly with the order, making the fourth and subsequent distortion orders almost negligible compared to the second and third. Simplifying the equations in Ref. 11 with the previous consideration, Eq. (1) is obtained:

$$
\left\{\begin{array}{l}
H_{1}(\omega)=H_{1}^{\prime}(\omega)+3 H_{3}^{\prime}(\omega), \\
H_{2}(\omega)=-2 \hat{H}_{2}^{\prime}(\omega), \\
H_{3}(\omega)=-4 H_{3}^{\prime}(\omega),
\end{array}\right.
$$

where $H_{1}^{\prime}, H_{2}^{\prime}, H_{3}^{\prime}$ are the first three harmonics of the impulse response $\left(H_{1}^{\prime}\right.$ the linear part and $H_{2}^{\prime}, H_{3}^{\prime}$ the two first distortion orders), and $H_{1}, H_{2}, H_{3}$ are the Diagonal Volterra kernels ( represents Hilbert transform).

Therefore, the second and third order non-linear distortions can be simulated by convolution, as shown in Eq. (2), where $x(n)$ is the input signal and $M$ is the number of samples of the kernel,

$$
\begin{aligned}
y(n)= & \sum_{i=0}^{M-1} h_{1}(i) \cdot x(n-i)+\sum_{i=0}^{M-1} h_{2}(i) \cdot x^{2}(n-i) \\
& +\sum_{i=0}^{M-1} h_{3}(i) \cdot x^{3}(n-i) .
\end{aligned}
$$

To simulate the linear part of the system response, only the first harmonic $H_{1}^{\prime}$ is employed (i.e., $H_{2}^{\prime}=H_{3}^{\prime}=0$ ). Applying this technique to a sound stimulus, it is possible to simulate the effect of a frequency response with and without the measured non-linear distortion.

\section{Subjective test}

The purpose of this test is to verify whether the distortion produced by each equalized headphone at different reproduction levels can be perceived or not. To avoid visual and tactile biases, all the different headphone emulations and their distortions measured were simulated through the reference headphone in a virtual simulation listening test. Wearing just the reference headphones, the subjects performing the test can have immediate access to the different headphones and the procedure of the test becomes more flexible, transparent, controlled, and repeatable. ${ }^{12}$ This methodology is desirable due to the differences in appearance, fitting, and range of qualities of the headphones employed.

The process of generating the stimuli for the virtual headphone listening test consists of using the method described in Sec. II B and applying the compensation filter of the reference headphone response. This filter was obtained with an automatic regularized method for the inversion of the frequency response, which produces perceptually better equalization than the regularized inverse method with a fixed factor. ${ }^{13}$ In this case, the mean of five repositioned measurements of the reference headphone response was used. 

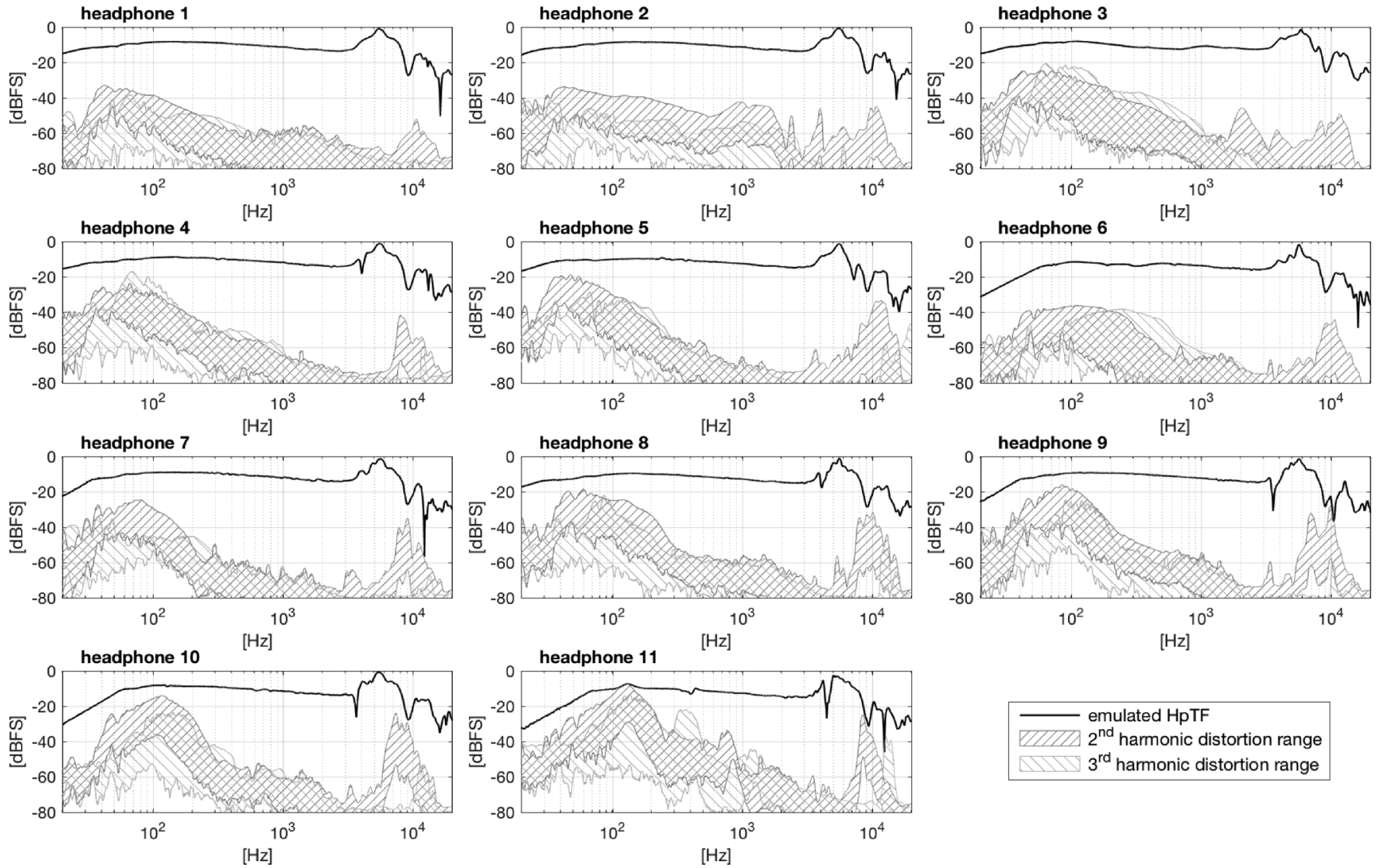

FIG. 1. Linear frequency responses achieved for each headphone after the emulation, and the first two non-linear distortion harmonics generated (range from minimum to maximum levels measured). Left channels.

To evaluate the perception of the non-linear distortion, the stimuli generated with and without distortion were presented to ten expert listeners ${ }^{14}$ by means of an $\mathrm{ABX}$ test. The different emulations achieved for each headphone were presented randomly. Positive detections of the distortion were considered at a significance level of $\alpha \leq 0.02 .{ }^{15}$ The sound clip employed to generate the stimuli of the test is accessible online. ${ }^{16}$ It was selected because of its rich low frequency content and large dynamic range. The reproduction level of the stimuli during the test was fixed at $85 \mathrm{dBA}$ $\left(\right.$ slow $\left.^{8}\right)$. With this ABX test, subjects identified the minimum level at which they detected the non-linear distortion for each of the emulated headphones. The number of stimuli generated were 11 headphones $\times 6$ levels $\times 2$ with-without distortion $=132$ stimuli. Absolute ecological validity is achieved just for the fixed reproduction level. This procedure has been employed in other works, ${ }^{17}$ suggesting that for the rest of the levels, if anything, this should result in an increased sensitivity of subjects to audible distortion.

\section{RESULTS}

The results of the ABX test can be seen in Fig. 2. The mean of the minimum reproduction levels with distortion detection is shown for each headphone. In addition, prices of the headphones were determined with the average of the retail price (\$USD) during the last 24 months. Thanks to this, headphone models are sorted by the retail price, with number 1 as the most expensive and 11 as the cheapest. A high correlation has been found between the minimum detection level of the distortion and the retail price $\left(r^{2}=0.91, p<0.001\right)$. Besides, three groups of headphones can be identified: Group A-headphones 1 and 2, priced above \$200; Group B-headphones 3-7, priced from \$200 to $\$ 20$; and Group $\mathrm{C}$ - -headphones $8-11$, priced at less than $\$ 20$. These groups have been found to correspond with different detected distortion levels: group A with an interval

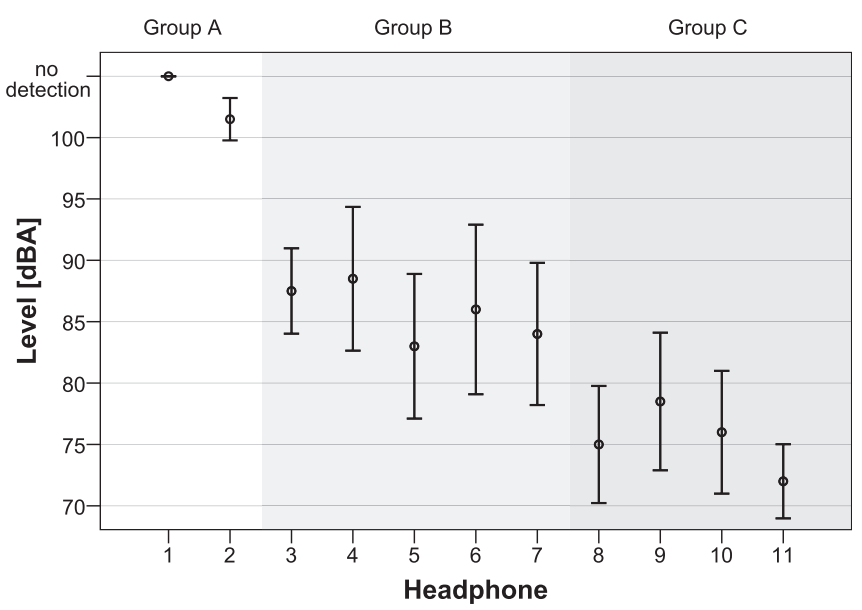

FIG. 2. Mean of the minimum reproduction levels with detected distortion for each headphone ( $95 \%$ confidence intervals). Headphone models are sorted by the retail price. 
from no distortion detected to some detections at $100 \mathrm{dBA}$, group $\mathrm{B}$ presenting a range of distortion detected from 95 $\mathrm{dBA}$ to $80 \mathrm{dBA}$, and group $\mathrm{C}$ having a range of distortion detected from around 80 to $70 \mathrm{dBA}$.

It is interesting to point out that in group $B$, most of the headphones have a pricing range from $\$ 100$ to $\$ 20$, except for headphone 3, which exceeds $\$ 100$. This model is a bit out of the distortion detection trend, with slightly worse results according to its price.

The main idea that emerges from these results is that for most of the headphones, the distortion is not noticeable at comfortable listening levels up to $80 \mathrm{dBA}$. Group A headphones with only a few detections at $100 \mathrm{dBA}$ can be considered to produce an almost undetectable distortion, as headphone users would rarely listen at levels over $95 \mathrm{dBA}$.

\section{CONCLUSIONS}

The emulation of a target frequency response over different headphone models can produce audible non-linear distortion, depending on the reproduction level.

In this study, a method has been implemented to measure and simulate the non-linear distortion produced by the emulation of a target frequency response (Sennheiser HD800) over diverse consumer headphones. This approach has allowed the frequency response achieved in the emulation to be simulated, with and without the non-linear distortions generated. Six different reproduction levels were analyzed on 11 headphone models.

An ABX test was performed by ten expert listeners to evaluate the audibility of the distortion generated at the different reproduction levels. High correlation has been found between the level of reproduction at which distortion is detected and the retail price of the headphones, with negligible detections in expensive models and a gradually increasing perception of the distortion as the price is reduced.

Some studies indicate that frequency response and the retail price of headphones have no correlation, ${ }^{1}$ but this experiment suggests that retail price can have a direct correlation with perceived non-linear distortion. Despite this, the frequency response equalization is shown to be a viable technique that does not produce disturbing distortion at moderate listening levels with medium quality headphones and not noticeable nonlinear distortion in the case of high-end headphones.

\section{ACKNOWLEDGMENTS}

The Spanish Ministry of Economy, Industry and Competitiveness supported this work under the project TEC2015-68076-R and Grant No. BES-2013-065034.

${ }^{1}$ J. Breebaart, "No correlation between headphone frequency response and retail price," J. Acoust. Soc. Am. 141(6), EL526-EL530 (2017).

${ }^{2}$ D. W. Martin and L. J. Anderson, "Headphone measurements and their interpretation," J. Acoust. Soc. Am. 19(1), 63-70 (1947).

${ }^{3} \mathrm{~F}$. Briolle and V. Thierry, "Transfer function and subjective quality of headphones: Part 2, Subjective quality evaluations," in Proceedings of the Audio Engineering Society 11th International Conference, Portland, OR (May 29-31, 1992), pp. 254-259.

${ }^{4}$ J. Backman, T. Campbell, J. Kleimola, and M. Hiipakka, "A SelfCalibrating Earphone," in Proceedings of the Audio Engineering Society 142nd Convention, Berlin, Germany (May 20-23, 2017).

${ }^{5}$ J. Liski, V. Välimäki, S. Vesa, and R. Väänänen, "Real-time adaptive equalization for headphone listening," in Proceedings of the 25th European Signal Processing Conference (EUSIPCO), Kos Island, Greece (August 28-September 2, 2017), pp. 638-642.

${ }^{6}$ T. Hirvonen, M. Vaalgamaa, J. Backman, and M. Karjalainen, "Listening test methodology for headphone evaluation," in Proceedings of the Audio Engineering Society 114th Convention, Amsterdam, the Netherlands (March 22-25, 2003).

${ }^{7} \mathrm{P}$. Gutierrez-Parera and J. J. Lopez, "Influence of the quality of consumer headphones in the perception of spatial audio," Appl. Sci. 6(4), 117 (2016).

${ }^{8}$ IEC 61672-1:2013: Electroacoustics-Sound Level Meters-Part 1: Specifications (IEC, Geneva, Switzerland, 2013).

${ }^{9}$ A. Novak, P. Lotton, and L. Simon, "Synchronized swept-sine: Theory, application and implementation," J. Audio Eng. Soc. 63(10), 786-798 (2015).

${ }^{10}$ A. Kulkarni and H. S. Colburn, "Variability in the characterization of the headphone transfer-function," J. Acoust. Soc. Am. 107(2), 1071-1074 (2000).

${ }^{11} \mathrm{~L}$. Tronchin, "The emulation of nonlinear time-invariant audio systems with memory by means of Volterra series," J. Audio Eng. Soc. 60(12), 984-995 (2012), available at http://www.aes.org/e-lib/browse.cfm?elib= 16639.

${ }^{12}$ S. E. Olive, T. Welti, and E. McMullin, "A virtual headphone listening test methodology," in Proceedings of the Audio Engineering Society 51st International Conference, Helsinki, Finland (August 21-24, 2013).

${ }^{13}$ J. Gómez Bolaños, A. Mäkivirta, and V. Pulkki, “Automatic regularization parameter for headphone transfer function inversion,” J. Audio Eng. Soc. 64(10), 752-761 (2016).

${ }^{14}$ ITU-RBS. 1116-3: Methods for the Subjective Assessment of Small Impairments in Audio Systems (ITU, Geneva, Switzerland, 2015).

${ }^{15}$ D. L. Clark, "Ten years of A/B/X testing," in Proceedings of the Audio Engineering Society 91 st Convention, New York (October 4-8, 1991).

${ }^{16}$ Dubstep loop $140 \mathrm{bpm}$ by waveplay, https://freesound.org/people/waveplay/sounds/198495/ (Last viewed September 25, 2017).

${ }^{17}$ S. Temme, S. E. Olive, S. Tatarunis, T. Welti, and E. Mcmullin, "The correlation between distortion audibility and listener preference in headphones," in Proceedings of the Audio Engineering Society 137th International Convention, Los Angeles, CA (October 9-12, 2014). 